\title{
PROTHROMBIN TIME IN DICOUMAROL THERAPY
}

\author{
BY \\ G. A. JAMES \\ E.M.S. Central Laboratory, Sector IV
}

(RECEIVED FOR PUBLICATION, JUNE, 1948)

In the course of controlling dicoumarol therapy given $(a)$ as a postoperative routine to prevent thrombosis (fifty-five cases) and (b) therapeutically in cases of established venous thrombosis (nine cases), certain peculiarities have been observed in the behaviour of the prothrombin time, particularly in relation to dicoumarol haemorrhage. Two sources of thromboplastin have been used, Russell viper venom and human brain. Data are presented which suggest that the brain extract is to be preferred to venom, for estimations of prothrombin levels are then more in accord with clinical observation.

\section{Technical Methods}

With Russell viper venom. - This method is essentially that of Fullerton's (1940) modification of Quick's one-stage method, with the slight additional modifications noted below:

$2 \mathrm{ml}$. of venous blood was placed in a tube containing $1.6 \mathrm{mg}$. of potassium oxalate and $2.4 \mathrm{mg}$. ammonium oxalate, shaken well, and centrifuged at 1,500 r.p.m. for 5 minutes, and the plasma is used in the test. Fullerton used liquid sodium oxalate, but providing that the quantity of b'ood put in the tube with the solid oxalate is not less than $1.5 \mathrm{ml}$. and not more than $2.5 \mathrm{ml}$. the results are the same.

The test $-0.1 \mathrm{ml}$. of oxalated plasma and $0.1 \mathrm{ml}$. of $1 / 10,000$ "Stypven" solution were placed in a tube in a $37^{\circ} \mathrm{C}$. water bath and left for 6 minutes (see below). $0.1 \mathrm{ml}$. of $\mathrm{M} / 40$ calcium ch'oride at $37^{\circ} \mathrm{C}$. was added quickly, and the time taken until the appearance of fibrin particles was noted. All estimations were performed in triplicate.

The tubes containing oxalated plasma and Stypven soiution were left for 6 minutes at $37^{\circ} \mathrm{C}$. because it was found that up to 4 or 5 minutes at $37^{\circ} \mathrm{C}$. the prothrombin time increased fairly rapidly, and reproducible resu'ts could not be obtained, while between 5 and 9 minutes little change occurred, and consequently results were more reliable. This change is probably due to two factors: (i) destruction of Quick's labile factor, and (ii) the proteolytic activity of the Stypven (Hobson and Witts, 1941).

With human brain extract.-The method is exactly the same as that described by Aggeler and others (1946). Points to be watched are: $(a)$ the brain extract must be very finely ground, as, if the particles are too large, they sett'e in the saline too rapidly, leaving too clear a supernatant fluid, which is almost devoid of thrombokinase activity-it appears that this activity resides in the particles themselves ; $(b)$ over-incubation of the brain extract in saline before use must be avoided- -12 minutes at $50^{\circ} \mathrm{C}$. is ample ; activity falls rapidly after 15 minutes at this temperature.

The prothrombin content of the plasma was read off on a graph of prothrombin time in seconds against percentage plasma concentration (in saline diluent).

The graphs used were prepared from the first patients in the series, twenty cases for the venom method, ten cases for Quick's. As the curves were all the same shape for the different patients, though shifted up or down the ordinate according to the individual variation, it was found possible, instead of preparing a fresh curve for each patient, to use one curve for the venom method and one for Quick's method, using parallel curves to allow for the variation.

General management of experiment.-Each case was tested before and after operation as a routine; dicoumarol was started on the fourth postoperative day $(300 \mathrm{mg}$. on the first evening, $200 \mathrm{mg}$. on the next); and in the morning after the two doses, counted as "day 2," the prothrombin time was estimated, and thereafter every other day until treatment was finished and the patient was ambulant. Further tests were carried out two or more days after cessation of therapy to see how quickly the prothrombin levels returned to normal. Patients requiring emergency operation or cases of established phlebothrombosis were tes!ed before treatment and thereafter in the same manner as above.

\section{Results}

Patients before operation.-It was observed that the prothrombin time of 55 patients before gynaecological operation varied between 10 and 21 seconds with the venom method. This variation is somewhat greater than has been found in previous investigations on normal subjects. For instance, Fullerton (1940) found a variation of 18 to 25 seconds, while Witts and Hobson (1942), using venom and lecithin, found one of 8 to 12 seconds. Aggeler and others (1946), in a full 
statistical study of 30 normal subjeets, using Quick's technique but with human brain extract, found a variation of only 10 to 13 seconds, whereas Nygaard (1941), also using Quick's technique but with rabbit brain extract, found a variation of 11 to 19 seconds.

Three subjects of the present series tested three or more times over a period of three to six months have remained constant to within 3 seconds of their original times, and Witts and Hobson suggested in their paper (1942) that the variations found in different individuals tested by the same method represented a true variation in the coagulability of normal plasmas:

\section{Patients under dicoumarol therapy}

USING RUSSELL VIPER VENOM.-Under dicoumarol therapy the prothrombin levels fell more or

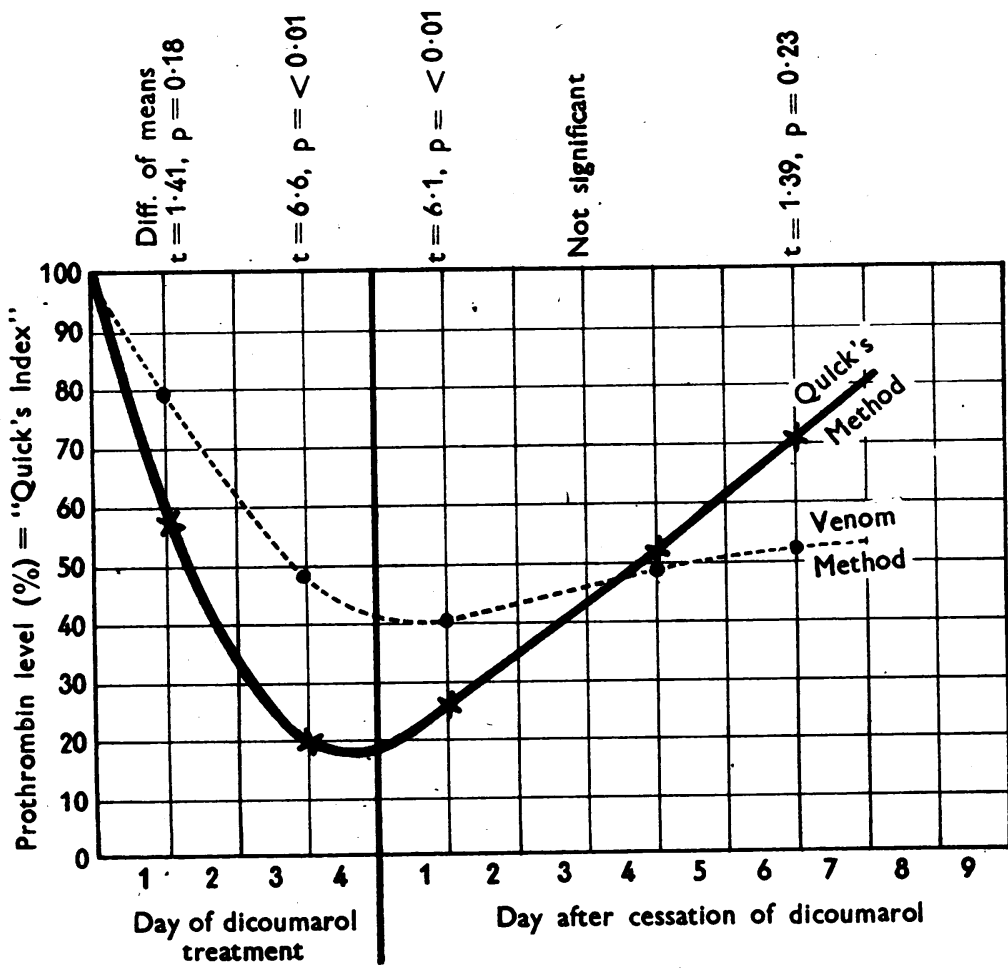

FIG. 1.-Graph showing response to dicoumarol of the prothrombin level as estimated by Fullerton's and by Quick's methods. Made from figures obtained from ten patients who had sufficient estimations and courses of dicoumarol of similar duration. All other cases showed exactly similar responses, but owing to clinical exigency such as variations in duration of treatment, etc., they could not be included in the construction of the graph. The differences between the readings on the graph are statistically significant at the point of inflexion of the curves, that is, after two days on dicoumarol and for three days after stopping the drug, when the curves approach each other. less as expocted, but it was soon found that patphitso were bleeding at levels higher than those usually regarded as dangerous. Quick has stated that the prothrombin level falls to below 10 per cent beforeo haemorrhage occurs, whereas in the first series ofo thirty cases controlled only by the venom method seven patients bled when their prothrombin levels $\mathbb{\Omega}$ were much higher than this-24 to 45 per cent.

Two patients developed severe vaginal haemorrhages and required transfusion. However, both $\odot$ had amputations of the cervix, after which it is almost impossible to obtain adequate covering of the raw area. On the other hand, several patientsos subjected to this operation have been treated with out resulting haemorrhage. Four patients bledfrom sites not involved in the operation.

It was noted that in half the cases the prothrom-0 bin levels continued to fall for 2 to 3 days even though the haemorrhage had ceased.

USING QUICK'S METHOD PARALLEL WITH THE VENOM METHOD.-In an endeavouis to find a more reliable method, Quick's technique was tried in conjunction withs the venom method on the blood of the next patienf who bled, and, as pointed out by Lempert (1948), the prothrombin level was found to be much lower as esti mated by Quick's methoफ़ than by the venom method.

In view of this, it was decided to do the two tests. in parallel on the next twenty cases. This has been done and the results in ten patients are summarized in Fig. 17 The ten cases not recordect. on this graph had had longefo courses of dicoumarol of had tests at less regulae intervals, but they all showed similar features.

The behaviour of the pro thrombin level.-In every? case, on and after the secondo day of dicoumarol adminise् tration the prothrombin leve $\vec{P}$ fell much more rapidit as estimated by Quick's? 
method than by the venom method, and in every case the prothrombin level returned towards normal far more rapidly (with due allowance for the lower level to which it had fallen) when estimated by Quick's method (Fig. 1).

Haemorrhages. - Three cases in this series had haemorrhages, all slight.

The prothrombin level of Case 33 had fallen by the seventh day to 8 per cent (Quick's) or 24 per cent (venom), and dicoumarol was accordingly stopped. She was well until the evening of the ninth day, when she complained of some pain in the loins, and next morning slight haematuria was noticed. This lasted only one day, and the next day her prothrombin level had risen by Quick's method to 20 per cent, while by the venom method it had shown no improvement, being 23 per cent, although the bleeding had ceased.

Case 41 had a sudden haemorrhage of $4 \mathrm{oz}$. from her abdominal wound on the ninth postoperative day, after only $700 \mathrm{mg}$. of dicoumarol. The blood appeared stale, and oozing of dark blood continued for some days. The clinician thought it was a haematoma. The patient's prothrombin level was 28 per cent by Quick's and 65 per cent by the venom method.

Case 55, who had had a vaginal hysterectomy, had slight vaginal haemorrhage on the ninth postoperative day, lasting for two days, and also some fever $\left(99.6^{\circ}\right.$ F.), both of which manifestations the surgeon thought were due to a mildly infected operation site. A slight oozing from the venipuncture hole suggested an overdose of dicoumarol. Her prothrombin level was 28 per cent by Quick's and 50 per cent by the venom method.

\section{Discussion}

In view of the more rapid fall in prothrombin level when estimated by Quick's method, less dicoumarol was required to keep the prothrombin level between 10 and 30 per cent, and consequently fewer haemorrhages occurred.
It may be argued that the levels given by the venom method are the correct ones, and that if one aimed, say, at a level of 40 to 60 per cent prothrombin adequate control could be obtained. However, in view of the slower response with the venom method, adequate warning of impending haemorrhage is not given and cases cannot be controlled so accurately.

The object of this treatment is to prevent thrombosis, and it may be argued that the smaller dosage of dicoumarol required to reduce the prothrombin level to 30 per cent using Quick's method gives insufficient protection against this danger. We have had one case of venous thrombosis in the latter series: in a woman who had a vaginal hysterectomy and was very septic, with fever and discharge. It occurred after $1,300 \mathrm{mg}$. dicoumarol, when her prothrombin level was about 40 per cent (Quick) and 35 per cent (venom) two days after cessation of dicoumarol therapy and when the patient was getting up. The thrombosis was a slight one, and with two doses of heparin and more dicoumarol no further trouble ensued. The correlation between prothrombin levels by the two methods, clinical findings, and dicoumarol administration in this case is seen in Fig. 2, which illustrates well the more lively response of Quick's method to administration or withdrawal of dicoumarol, and the value of daily estimations of prothrombin time in controlling treatment.

Though the venom methods of prothrombintime estimation may. give moderately reliable figures on normal plasmas, they do not follow the true prothrombin level (or at least the blood coagulability) sufficiently closely to give warning of impending haemorrhage, and the methods using acetone dried brain extract as a thromboplastin are far more reliable in this respect. In fact there

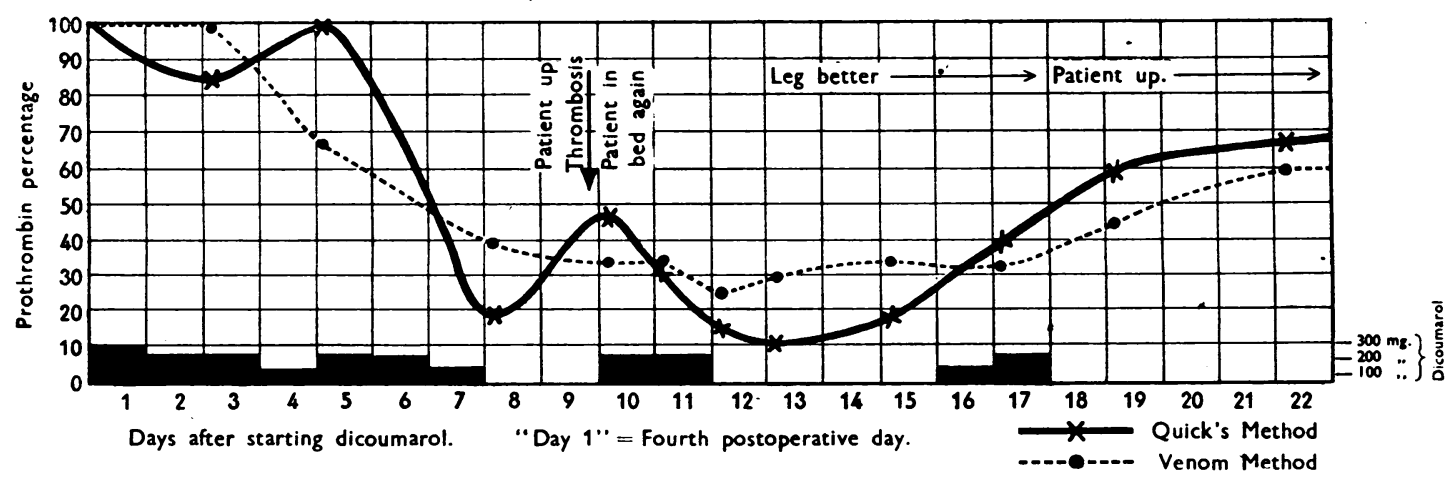

FIG. 2.-Graph of the prothrombin level of Case 53, showing time-relations of dicoumarol administration, thrombosis, etc., to changes in prothrombin level as measured by Quick's and Fullerton's methods. 
seems to be a delay of two or three days in the prolongation of prothrombin time as estimated by the venom method, so that prolongation occurs after the haemorrhage has taken place; and in some cases there is very little prolongation even though severe haemorrhage has occurred.

Neither of these serious defects has been observed with the brain extract method, so that, for the safety of the patients and the peace of mind of the pathologist, a return to Quick's method seems long overdue.

\section{Summary}

While controlling the treatment of patients on dicoumarol with Russell viper venom method of prothrombin-time estimation, it was noted that some patients bled with prothrombin concentrations anywhere between 24 and 45 per cent.

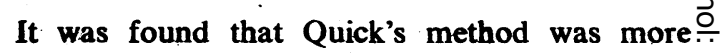
sensitive to changes in prothrombin in patients on $\overrightarrow{\overrightarrow{\mid}}$ dicoumarol, and that it provided a more accurate forecast of possible haemorrhage. A return to this method is advocated.

I wish to express my thanks to Dr. Schwabacher $\overparen{\Phi}$ for the interest and help she has shown throughout. to Dr. J. V. Dacie for his kindly and constructive criticism, to Dr. Reid for checking the statistics, to Mr. Fincham and Miss Taylor for their technical assistance, and lastly to Professor Browne, upon $\vec{A}$ whose patients these investigations were carried out.

\section{REFERENCES}

Aggeler, P. M., Howard, J., Lucia, S. P., Clark, W., and Astaff, A. (1946). Blood, 1, 224

Fullerton, H. W. (1940). Lancet, 2, 195 Fullerton, H. W. (1940). Lancet, 2, 195.
Lempon, F. C. G., and Witts, L. J. (1941). J. Path. Bact., 52, 367.

Nygaard, K. K. (1941). "Haemorrhagic Diseases." St. Louis.

Quick, A. J. (1936). Amer. J. Physiol., 115, 317.

Quick, A. J. (1938). J. Amer. med. Ass., 110, 1658.

Quick, A. J. (1940). Amer. J. clin. Path., 10, 228.

Quick, A. J. (1940). Amer. J. clin. Path., 10, 228.

Witts, L. J., and Hobson, F. C. G. (1940). Brit. med. J., 2, 862

Witts, L. J., and Hobson, F. C. G. (1942). Brit. med. J., 1, 575 